\title{
Aldesleukin Regimen
}

National Cancer Institute

\section{Source}

National Cancer Institute. Aldesleukin Regimen. NCI Thesaurus. Code C160804.

A regimen consisting of aldesleukin that may be used in the treatment of metastatic melanoma and metastatic renal cell carcinoma (RCC). 